CORRIGENDUM

\title{
Bracing for the biosimilar wave
}

Asher Mullard

Nature Reviews Drug Discovery 16, 152-154 (2017)

The article has been updated to indicate that Sandoz ran a confirmatory study in chronic plaque psoriasis of its etanercept biosimilar versus active comparator, rather than versus placebo. 